\title{
Correction to: A Nonsense N -Terminus NFKB2 Mutation Leading to Haploinsufficiency in a Patient with a Predominantly Antibody Deficiency
}

Hye Sun Kuehn ${ }^{1}$ - Andrea Bernasconi ${ }^{2}$. Julie E. Niemela ${ }^{1}$. Maria Belen Almejun ${ }^{2}$ - William Alexander Franco Gallego ${ }^{3}$. Shubham Goel ${ }^{1}$ • Jennifer L. Stoddard ${ }^{1}$ • Ronald Guillermo Peláez Sánchez ${ }^{3}$. Carlos Andrés Arango Franco ${ }^{3}$. Matías Oleastro $^{2}$ - Eyal Grunebaum ${ }^{4}$ - Zuhair Ballas ${ }^{5}$. Charlotte Cunningham-Rundles ${ }^{6}$. Thomas A. Fleisher ${ }^{1}$. José Luis Franco ${ }^{3}$ Silvia Danielian ${ }^{2} \cdot$ Sergio D. Rosenzweig ${ }^{1}$

Published online: 9 September 2020

(C) Springer Science+Business Media, LLC, part of Springer Nature 2020

Correction to: Journal of Clinical Immunology https://doi.org/10.1007/s10875-020-00842-2

Due to typesetting mistake, the caption of Figure 2 was mistakenly replaced with the caption of Figure 3.

The original version has been corrected.

Publisher's Note Springer Nature remains neutral with regard to jurisdictional claims in published maps and institutional affiliations.

The online version of the original article can be found at https://doi.org/ 10.1007/s10875-020-00842-2

Hye Sun Kuehn

hyesun.kuehn@nih.gov

$\triangle$ Sergio D. Rosenzweig

srosenzweig@cc.nih.gov

1 Immunology Service, Department of Laboratory Medicine, Clinical Center, NIH, Building 10, Room 2C306, 10 Center Drive,

MSC1508, Bethesda, MD, USA

2 Department of Immunology, "Juan P. Garrahan" National Hospital of Pediatrics, Buenos Aires, Argentina

3 Grupo de Inmunodeficiencias Primarias, Universidad de Antioquia, UDEA, Medellín, Colombia

4 Division of Immunology and Allergy, Department of Pediatrics, Hospital for Sick Children, Toronto, ON, Canada

5 Department of Internal Medicine, University of Iowa Carver College of Medicine, Iowa City, IA, USA

6 Division of Clinical Immunology, Department of Medicine, Icahn School of Medicine at Mount Sinai, New York, NY, USA 